\title{
Analysis on Family History of Diabetes, Weight Gain during Pregnancy and Pre-pregnancy Body Mass Index on 82 Pregnant Women with Gestational Diabetes Mellitus
}

\author{
Jingyun Gao, Zhaozhao Hua*, Anqin Wu \\ The Second Affiliated Hospital of Guizhou University of Traditional Chinese Medicine, Guiyang 550002, Guizhou Province,
} China

*Corresponding author: Zhaozhao Hua, 859245757@qq.com

Copyright: (C) 2022 Author(s). This is an open-access article distributed under the terms of the Creative Commons Attribution License (CC BY 4.0), permitting distribution and reproduction in any medium, provided the original work is cited.

\begin{abstract}
Objective: To investigate the effects of family history of diabetes mellitus, Gestational Weight Gain (GWG) and Body Mass Index (BMI) before pregnancy on Gestational Diabetes Mellitus (GDM). Method: 82 pregnant women with GDM who were hospitalized and delivered in the obstetrics department of our hospital from September 2017 to September 2019 were selected as the observation group, and 60 pregnant women with normal glucose tolerance test in the same period were selected as the control group; The relationship between family history of diabetes, weight gain during pregnancy and prepregnancy Body Mass Index and GDM were analyzed. Results: The age, pre-pregnancy weight and weight gain during pregnancy were significantly higher in the observation group than in the control group $(\mathrm{P}<0.05)$, and the family history of diabetes and pre-pregnancy Body Mass Index were higher in the observation group than in the control group $(\mathrm{P}<0.05)$, and the differences were statistically significant. Conclusion: It is suggested that family history of diabetes is related to gestational diabetes mellitus. Excessive GWG growth during pregnancy and high Body Mass Index before pregnancy may increase the risk of gestational diabetes mellitus in pregnant women.
\end{abstract}

Keywords: Gestational diabetes mellitus; Family history of diabetes; Weight gain during pregnancy; Body Mass Index

Online publication: January 19, 2022

\section{Introduction}

The incidence of Gestational Diabetes Mellitus (GDM) is increasing day by day, which affects pregnant women by $5 \%-15 \%$. To a certain extent, the incidence of delivery complications in this series of pregnant women is also increasing, for example: cesarean section, shoulder dystocia, fetal macrosomia, and postpartum hemorrhage ${ }^{[1]}$. Also after childbirth, the risk of gestational diabetes continues to threaten these women. Studies have shown that women with a previous history of gestational diabetes were nearly eight times more likely to develop type 2 diabetes in the future ${ }^{[2]}$, were also more likely to have gestational diabetes in a second pregnancy, and were more likely to have type 2 diabetes in both their partners and their offspring ${ }^{[3]}$. The purpose of this study is to explore the effects of family history of diabetes, weight gain during pregnancy and pre-pregnancy BMI on gestational diabetes mellitus, so as to provide some help and consideration for clinical perinatal maternal and child health care. 


\section{Materials and methods}

\subsection{General information}

A total of 82 pregnant women with gestational diabetes mellitus who were hospitalized in the Second Affiliated Hospital of Guizhou University of Traditional Chinese Medicine from September 2017 to September 2019 were selected as the observation group, and the data of pre-pregnancy Body Mass Index and weight gain during pregnancy were collected. 60 pregnant women with normal gestational glucose tolerance test (24-28 weeks of pregnancy) were selected as the control group. The age of the observation group was 22-37 years old, with an average of (29.06 \pm 3.13$)$ years old; There were 19 multiparas and 63 primiparas. The age of the control group was 23-35 years old, with an average of (27.85 \pm 4.15$)$ years old; There were 22 multiparas and 38 primiparas.

\subsection{Inclusion and exclusion criteria}

\subsubsection{Inclusion criteria}

(1) In line with all diagnostic criteria of 2014 "Guidelines for Diagnosis and Treatment of Gestational Diabetes mellitus" [4].

(2) OGTT of 75g glucose powder was completed to diagnose gestational diabetes mellitus at 24-28 weeks of gestation. All the selected patients had a single pregnancy, and were hospitalized for delivery for 6 days from 37 weeks to 41 weeks.

(3) There were no cardiovascular diseases such as heart disease and hypertension before pregnancy.

(4) They didn't have diabetes, thyroid disease, other endocrine system diseases, and a history of chronic kidney disease.

\subsubsection{Exclusion criteria}

(1) Complicated with internal and external diseases.

(2) Smoking and drinking history.

(3) There has been a history of using salt, glucocorticoid drugs or drugs affecting lipid metabolism in recent six months.

\subsection{Index evaluation}

The pre-pregnancy weight was recorded, the BMI before pregnancy was calculated, and the family history of diabetes was counted. Blood samples were collected from twenty-fourth weeks to twenty-eighth weeks of pregnancy. Fasting venous blood was taken to measure and record fasting blood glucose, 1 hour postprandial blood glucose, and 2 hours postprandial blood glucose.

\subsection{Data analysis}

SPSS 22.0 was used for data analysis and processing. The measurement data was expressed as " $\bar{x} \pm \mathrm{s}$." $\mathrm{X}^{2}$ test was used for comparison, t-test of non-independent samples was used for comparison among groups, and paired t-test was used for comparison within groups; The count data was expressed in $\%$, and $\mathrm{P}<0.05$, indicating that the difference was statistically significant.

\section{Results}

\subsection{Comparison of general information}

(1) The age, pre-pregnancy weight and weight gain during pregnancy were significantly higher in the observation group than in the control group $(\mathrm{P}<0.05)$, and the differences were statistically significant.

(2) There was no significant difference in the proportion of parturient women between the two groups, which does not have statistical significance $(\mathrm{P}>0.05)$; As shown in Table 1. 
Table 1. Comparison of general data $(\overline{\mathrm{x}} \pm \mathrm{s})$

\begin{tabular}{lllll}
\hline Project & Observation group $\mathbf{n}=\mathbf{8 2}$ & Control group $\mathbf{n}=\mathbf{6 0}$ & $\mathbf{X}^{\mathbf{2}} / \mathbf{t}$ & $\mathbf{P}$ \\
\hline Age (years old, $\mathrm{x} \pm \mathrm{s}$ ) & $29.06 \pm 3.13$ & $27.85 \pm 4.15$ & 3.412 & 0.001 \\
Pre pregnancy weight & $56.72 \pm 7.87$ & $54.08 \pm 7.02$ & 2.803 & 0.039 \\
Multipara (cases, \%) & $19(23.17)$ & $22(36.6)$ & & 0.681 \\
Weight gain during pregnancy & $19.2 \pm 4.1$ & $14.3 \pm 4.0$ & 0.003 \\
\hline
\end{tabular}

\subsection{Compared with family history of diabetes mellitus and pre-pregnancy BMI}

The observation group was higher than the control group $(\mathrm{P}<0.05)$, and the difference was statistically significant. The BMI before pregnancy is positively correlated with the incidence of gestational diabetes mellitus. It also indicates that the higher the pre-pregnancy Body Mass Index is, the higher the incidence of gestational diabetes mellitus is $(\mathrm{P}<0.5)$.

Table 2. Comparison of DM family history and pre-pregnancy BMI $(\overline{\mathrm{x}} \pm \mathrm{s})$

\begin{tabular}{lllll}
\hline Project & Observation group $\mathbf{n}=\mathbf{8 2}$ & Control group $\mathbf{n}=\mathbf{6 0}$ & $\mathbf{X}^{\mathbf{2}} / \mathbf{t}$ & $\mathbf{P}$ \\
\hline Pre-pregnancy Body Mass Index & $25.3 \pm 1.7$ & $22.1 \pm 2.4$ & 10.911 & 0.004 \\
Family history of diabetes & $60(73.17)$ & $25(41.66)$ & & 0.003 \\
\hline
\end{tabular}

\section{Discussion}

The data of family history of diabetes showed that the observation group was higher than the control group ( $\mathrm{P}<0.05)$, and the difference was statistically significant. Therefore, it can be concluded that family history of diabetes has a certain relationship with the occurrence of gestational diabetes. As a result, medical history should be thoroughly checked clinically for pregnant women who are establishing maternal health cards for the first time. It also needs to centrally manage pregnant women with diabetes histories and actively guide their pre-pregnancy and pregnancy diet structures to reduce the risk of gestational diabetes, which can improve perinatal maternal and infant outcomes, reduce the series of long-term complications caused by gestational diabetes to some extent, and break the vicious cycle of poor metabolic memory passed down from mother to son. As a result, to promote healthy individuals while also reducing the fiscal load of modern society. Since this paper's data sample size is tiny, the research findings have certain limitations.

Those who are clearly obese prior to pregnancy (with a high BMI) and those who gain weight too quickly during pregnancy are both at risk for gestational diabetes. The blood glucose level of patients with gestational diabetes mellitus is significantly connected to pre-pregnancy obesity and pre-pregnancy BMI. As these two factors rise, the chance of gestational diabetes rises as well. In the future, women with gestational diabetes mellitus will be a high-risk category for diabetes. Some individuals who were unable to maintain their food and weight throughout pregnancy and postpartum have yet to regain normal glucose metabolism. The mother's body undergoes changes in glucose and lipid metabolism during the pregnancy in order to meet fetal growth and development as well as changes in hormone levels, which are mostly represented in an increase in lipid metabolism and a decrease in glucose tolerance. To some degree, an increase in blood lipid levels can fulfil the demands of fetal growth and development, but an imbalance of obesity and blood lipid levels in the maternal body can create metabolic disturbance, which may lead to gestational diabetes mellitus. The metabolism of glycolipids will interact and encourage one another. Insulin resistance in pregnant women with gestational diabetes mellitus appears to be more severe than in normal pregnant women, resulting in aberrant lipid metabolism ${ }^{[5]}$. Epidemiological studies show that the prevalence of obesity in pregnant women with diabetes in China has reached $37.9 \%{ }^{[6]}$. Studies have 
confirmed that almost all gestational diabetes mellitus patients have high body mass index before pregnancy and obvious weight gain before pregnancy ${ }^{[5]}$. Studies show that controlling diet and reasonable weight control can help to control blood glucose, and reduce the incidence of gestational diabetes mellitus ${ }^{[7]}$. Additionally, controlling the pre-pregnancy and pregnancy body mass index can improve the pregnancy outcome of women with gestational diabetes mellitus, which can reduce the incidence of macrosomia, the incidence of low-birth-weight infants, the incidence of cesarean section, and the incidence of complications such as gestational hypertension. Therefore, the family history of pregnant women should be made clear during the first prenatal examination, and positive and effective intervention should be carried out for weight gain before pregnancy and during pregnancy in high-risk patients. These practices can reduce the occurrence of gestational diabetes to a certain extent, reduce the probability of postpartum diabetes, and further improve the outcome of pregnancy and the fetus, so as to ensure the safety of perinatal mothers and infants, ensure responsibility, and do a good job in clinical work for the health of women and children.

\section{Disclosure statement}

The author declares no conflict of interest.

\section{References}

[1] Luat NC, Minh PN, Binns CW, et al., 2018, Prevalence of Gestational Diabetes Mellitus in Eastern and Southeastern Asia: A Systematic Review and Meta-Analysis. Journal of Diabetes Research, 1-10.

[2] Song C, Lyu Y, Li C, et al., 2018, Long-Term Risk of Diabetes in Women at Varying Durations After Gestational Diabetes: A Systematic Review and Meta-Analysis with More Than 2 Million Women. Obesity Reviews, 19(3): 421-429.

[3] Liu Y, Zhu L, Huang Y, et al., 2018, Effects of Different Pre-pregnancy BMI and Pregnancy BMI Increase on Pregnancy Outcome. Jilin Medical Journal, 039(003): 423-425.

[4] Obstetrics and Gynecology Branch of Chinese Medical Association, 2014, Pregnancy and Diabetes Association Group of Chinese Medical Association Perinatal Medicine Branch. Guidance on Diagnosis and Treatment of Gestational Diabetes Mellitus. Chinese Journal of Obstetrics and Gynecology, 49(8).

[5] Sun Z, Xu Y, Yang H, et al., 2019, Relationship between Pre-pregnancy Body Mass Index, Gestational Weight Gain and Gestational Glycolipid Metabolism in Gestational Diabetes Mellitus. Chinese Journal of Family Planning, 27(10): 1312-1316.

[6] Liu T, Li J, Zhou Y, et al., 2015, Epidemiological Investigation of Gestational diabetes Mellitus. Maternal and Child Health Care of China, 030(009): 1408-1410.

[7] Christopher KH, 2020, The New Gestational Diabetes: Treatment, Evidence. Aust N Z J Obstet Gynaecol, 60: 482-485.

Publisher's note

Bio-Byword Scientific Publishing remains neutral with regard to jurisdictional claims in published maps and institutional affiliations. 